patients, Org39141-treated patients showed a trend towards treatment efficacy. These results are encouraging for further clinical development of Org38141.

Original article Zandbelt MM et al. (2006) Intranasal administration of recombinant human cartilage glycoprotein-39. A phase I escalating cohort study in patients with rheumatoid arthritis. J Rheumatol 33: 1726-1733

\section{Dramatic ethnic differences in UK prevalence of lupus nephritis}

Estimates of the prevalence and incidence of lupus nephritis in the UK have revealed striking ethnic variation: white patients with systemic lupus erythematosus (SLE) had the lowest prevalence of lupus nephritis, followed (in ascending order) by Indo-Asian, Afro-Caribbean, and Chinese patients. Lupus nephritis is a major complication of SLE, with substantial morbidity and mortality; this study supports previous findings that SLE is not only more common, but also has a worse prognosis, in nonwhite compared with white ethnic groups.

Patel and colleagues identified 208 individuals (176 women) with biopsy-proven lupus nephritis in the northwest of England during 2001. Data from the 2001 census indicated a prevalence of lupus nephritis of 4.4 cases per 100,000 of the population. The prevalence and incidence of lupus nephritis were markedly higher in women than in men (prevalence, 7.1 versus 1.4 cases per 100,000; incidence, 0.68 versus 0.09 cases per 100,000 ), a pattern repeated in all ethnic subgroups; however, more men had lupus nephritis than expected. Patel et al. suggest that male sex (in patients with SLE) confers an increased risk of lupus nephritis. The prevalence of lupus nephritis was highest in Chinese patients (65.5 cases per $100,000)$, although only 13 Chinese patients were included in the study.

Notably, peak prevalence of lupus nephritis occurred earlier in Afro-Caribbean and Chinese women than in white and Indo-Asian women (at age 20-39 years, versus $40-59$ years). In men, prevalence increased with advancing age in all ethnic groups.

Patel and colleagues estimate that $10 \%$ of white patients, $27 \%$ of Indo-Asian patients, and $58 \%$ of Afro-Caribbean patients with SLE develop lupus nephritis.

Original article Patel M et al. (2006) The prevalence and incidence of biopsy-proven lupus nephritis in the UK: evidence of an ethnic gradient. Arthritis Rheum 54: 2963-2969

\section{Role of MRI of the hands in the early diagnosis of RA}

Early diagnosis and treatment of rheumatoid arthritis (RA) is important if patients are to have the best possible prognosis. There is, therefore, a need for improved detection of early RA in patients with undifferentiated polyarthritis who do not show radiographically visible erosive changes, and who do not have antibodies to cyclic citrullinated peptides (anti-CCP antibodies; these are associated with severe RA).

In this prospective study, 30 patients with inflammatory polyarthralgia or synovitis of at least one joint underwent radiography of their hands, wrists, and feet, and MRI of their hands. Axial MRI views of carpal and metacarpal joints were scored according to the Outcome Measures in Rheumatology system. All patients in the study were negative for antibodies to CCP and had normal baseline radiographs. At 1-year follow-up, 16 patients were diagnosed with RA.

Baseline MRI scores for carpal erosion, synovitis, and tenosynovitis did not differ between patients diagnosed with and without RA. There was, however, a statistically significant difference between these two groups in MRI scores for metacarpophalangeal joint erosion (19.3 versus 7.7 , respectively; $P=0.024$ ), particularly in scores of the second and third metacarpophalangeal joints. Analysis showed that an MRI score >15 for metacarpophalangeal joint erosion had a specificity of $70 \%$ and a sensitivity of $64 \%$ for the prediction of RA.

Solau-Gervais and colleagues suggest that MRI scores for metacarpophalangeal joint erosion could be a useful tool in the early diagnosis of RA, especially in patients negative for antibodies to CCP.

Original article Solau-Gervais E et al. (2006) Magnetic resonance imaging of the hand for the diagnosis of rheumatoid arthritis in the absence of anti-cyclic citrullinated peptide antibodies: a prospective study. J Rheumatol 33: 1760-1765 